\title{
Diagnóstico estructural de un edificio de hormigón armado basado en su perfil bio-sísmico y un análisis dinámico incremental
}

\author{
Structural diagnosis of a RC building based on its bio-seismic profile and an incremental \\ dynamic analysis
}

Fecha de entrega: 24 de septiembre 2019

Fecha de aceptación: 19 de marzo 2020

\section{Juan J. Olivera-López y Claudio A. Oyarzo-Vera}

Departamento de Ingeniería Civil, Facultad de Ingeniería, Universidad Católica de la Santísima Concepción, Alonso de Ribera 2850, Casilla 297, Concepción, Chile, jolivera@magister.ucsc.cl, coyarzov@ucsc.cl

En este estudio se presenta la evaluación global de la salud estructural de un edificio habitacional de 14 pisos de altura, potencialmente vulnerable a sufrir daños significativos debido a terremotos y tsunamis. Este edificio fue construido en el año 2013 y está ubicado en la zona costera de Concepción (Chile), en el área afectada por el terremoto $M_{w}=8.8 \mathrm{del}$ Maule 2010. La evaluación estructural se realizó combinando ensayos destructivos (extracción y ensayo de testigos) $y$ no-destructivos (esclerómetro). Utilizando la información obtenida experimentalmente se generó un modelo numérico lineal del edificio, el cual fue utilizado para evaluar la salud global del edificio mediante una metodología llamada perfil bio-sísmico y un análisis dinámico incremental. Los resultados de este estudio demuestran que el edificio presentaria un buen desempeño frente a eventos sísmicos de gran envergadura, pero sería susceptible a presentar daño producto de los desplazamientos que experimentaría, ya que estos superan el rango de desplazamiento permitido por las juntas de expansión sísmica.

Palabras clave: perfil bio-sísmico, análisis dinámico incremental, evaluación estructural
This study presents a global structural health assessment of a 14-story high residential building, potentially vulnerable to damage due to earthquakes and tsunamis. This building was built in 2013 and is located in the coastal area of Concepcion (Chile), the same area affected by the $M_{w}=8.8$ earthquake in Maule 2010. The structural assessment was carried out by combining destructive tests (cores'extraction and test) and non-destructive tests (sclerometer). Using the information obtained experimentally, a linear numerical model of the building was generated, which was used to assess the overall health of the building using a methodology called bio-seismic profile and an incremental dynamic analysis. The results of this study demonstrate that the building would perform well during seismic events, but it would be susceptible to damage due to the displacements, since these exceed the range allowed by seismic expansion joints.

Keywords: bio-seismic profile, incremental dynamic analysis, structural assessment

\section{Introducción}

El fácil acceso a software de análisis estructural que se utiliza en el diseño de edificios, ha permitido la creación de diseños cada vez más audaces y complejos, lo que muchas veces dificulta prever de manera intuitiva su comportamiento ante solicitaciones sísmicas extremas. Esto hace que sea muy difícil identificar de manera temprana potenciales debilidades en el diseño. Además, muy pocas veces se implementan planes de monitoreo estructural que permitan contrastar el comportamiento real de la estructura con aquel estimado por las modelaciones numéricas (Ventura y Horyna, 1997; Peeters y Ventura, 2003; Wilson et al., 2008). Estas diferencias se originan, por lo general, debido a la incertidumbre que existe respecto a las propiedades mecánicas de los materiales, como por ejemplo la densidad, módulo de elasticidad, coeficiente de Poisson y módulo de corte del hormigón. Usualmente, los valores de las propiedades mecánicas utilizadas para el análisis y diseño provienen de análisis estadísticos, y entregan a lo sumo una buena aproximación del valor real. Sin embargo, estos valores pueden variar significativamente si no se tiene un buen control de calidad de los materiales durante la construcción de la obra. Otra fuente de estas 
diferencias dice relación con las condiciones de apoyo que proporciona el suelo de fundación, el comportamiento de las uniones entre los diferentes elementos y el efecto de los elementos no estructurales, todos ellos muy lejos de cumplir las condiciones ideales usualmente consideradas en la modelación.

En la actualidad, existen diversos métodos que permiten caracterizar el comportamiento de una estructura, algunos de naturaleza destructiva y otros menos invasivos. Dentro de los métodos destructivos se encuentran, por ejemplo, la extracción de testigos para la realización de ensayos de compresión, pero por lo general la cantidad de muestras que se pueden tomar de una estructura es limitada y sólo representativa de puntos y elementos específicos. Por otro lado, dentro de las técnicas no destructivas, se encuentran aquellas basadas en métodos indirectos, tales como los ensayos de esclerometría, que permiten determinar la resistencia del hormigón, y otros asociados a pruebas de vibraciones, como por ejemplo, el análisis modal, que da pistas respecto al comportamiento global de la estructura.

Los parámetros obtenidos mediante los métodos mencionados anteriormente sirven para ajustar los modelos numéricos utilizados durante la etapa de diseño, de manera que estos reflejen de mejor manera el comportamiento real de la estructura y en base a estos se puedan hacer mejores estimaciones de su comportamiento futuro.

El estudio que aquí se presenta tiene como objetivo diagnosticar la salud estructural de un edificio residencial de hormigón armado. Esta evaluación combina el uso limitado de extracción y ensayo de testigos con ensayos nodestructivos tales como pruebas de resistencia de hormigón mediante el uso del esclerómetro. Esto con el fin de ajustar un modelo numérico de la estructura, y así poder evaluar la salud global del edificio en base a una metodología conocida como perfil bio-sísmico (Guendelman et al., 1997) y un análisis dinámico incremental IDA (Vamvatsikos y Cornell, 2002).

\section{Descripción de la estructura y modelo numérico}

La estructura en estudio corresponde a un edificio residencial de 14 pisos construido en el año 2013 en la zona costera del Gran Concepción, Chile (Figura 1). Por motivos de acuerdos de confidencialidad con los residentes del edificio no se pueden proporcionar más datos o imágenes que permitan identificar esta construcción. El edificio está estructurado con marcos y muros de hormigón armado de 15, 20 y $25 \mathrm{~cm}$ de espesor. Las losas del edificio son de hormigón armado de $15 \mathrm{~cm}$ de espesor, lo que provee diafragmas rígidos que distribuyen las solicitaciones sísmicas a los elementos verticales. El edificio está dividido en cuatro módulos (A, B, C y D) separados por juntas de expansión sísmicas de $2.5 \mathrm{~cm}$ rellenas con poliestireno expandido (designadas como JD en la figura). El hormigón considerado para el diseño del edificio es de calidad G-25, es decir, $f^{\prime}{ }_{\mathrm{c}}=25 \mathrm{MPa}(\mathrm{NCh} 170,2016)$.
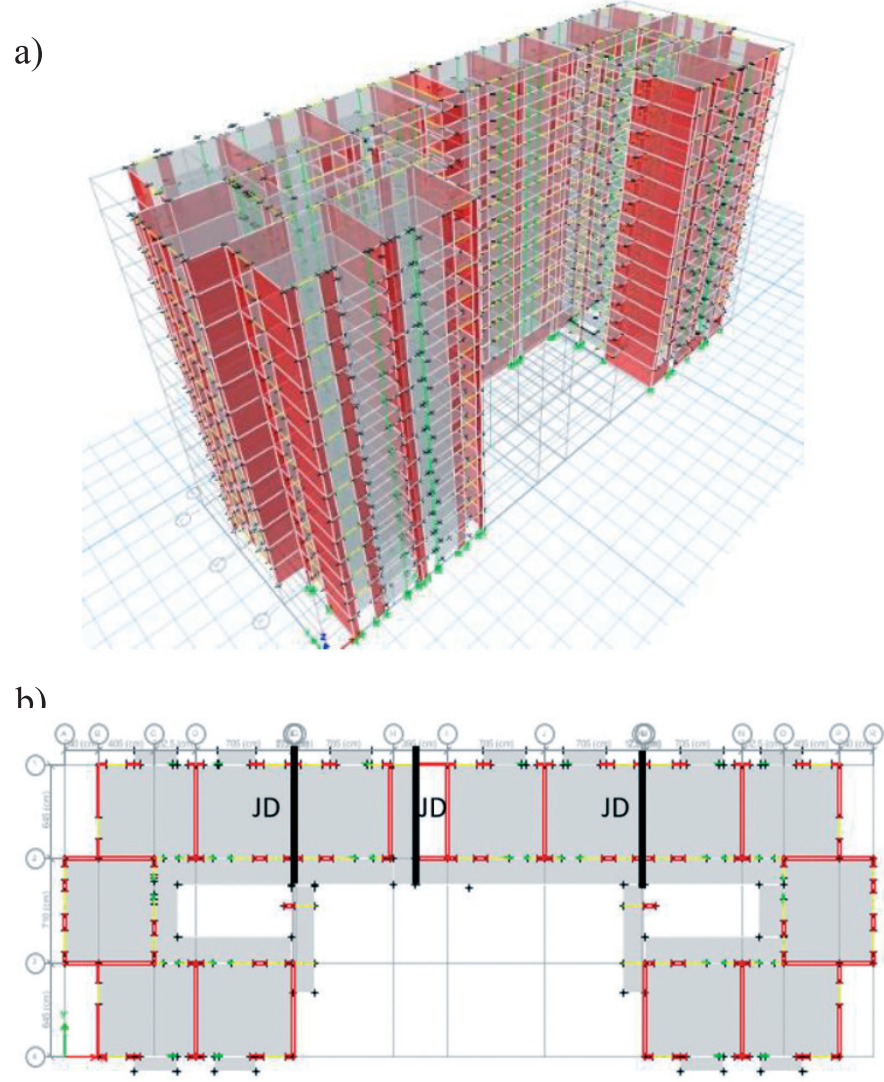

Figura 1: a) Vista 3D y b) vista en planta del edificio

\section{Determinación experimental de las propiedades del hormigón}

Con el fin de verificar si el hormigón utilizado en la construcción del edificio cumple con las especificaciones de diseño, se realizaron mediciones de su resistencia utilizando un esclerómetro. El esclerómetro, también conocido como Martillo Schmidt, es un dispositivo de prueba que permite determinar la resistencia del hormigón 
a través de mediciones de su dureza superficial, como se muestra en la Figura 2a (NCh1565, 2009). La adquisición de datos se realizó en 260 diferentes puntos de la losa en diferentes pisos del edificio (260 ensayos). En cada punto se realizaron 12 mediciones (ASTMC805, 2018) a fin de obtener un valor promedio de la dureza del hormigón (Figura 2b), descartando un máximo de dos mediciones cuando se alejan de la media $(\mathrm{CoV}>10 \%)$.

a)
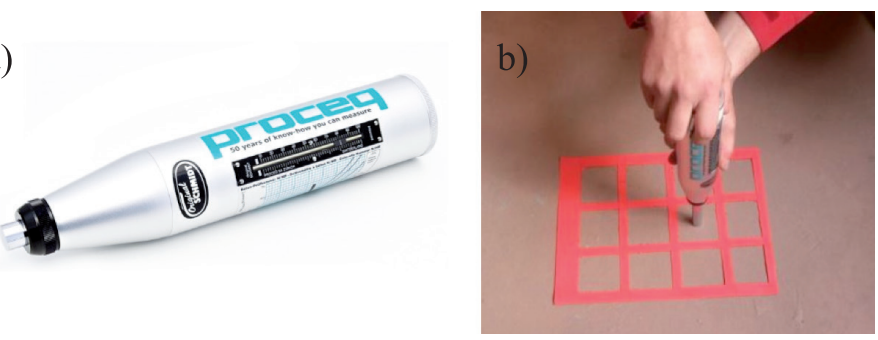

Figura 2: a) Martillo esclerométrico y b) proceso de adquisición de datos

En forma complementaria, se extrajeron dos testigos de hormigón (Figura 3) siguiendo el procedimiento especificado en la norma chilena NCh1171 (2012). Estos testigos fueron sometidos a ensayos de compresión para obtener la resistencia característica del hormigón $\left(f^{\prime}{ }_{c}\right)$ según el procedimiento estipulado en la norma chilena NCh1037 (2009). Con los resultados obtenidos del ensayo con esclerómetro y los ensayos de compresión del hormigón se obtuvo la clasificación del hormigón de cada piso según su resistencia tal como se muestra en la Tabla 1.

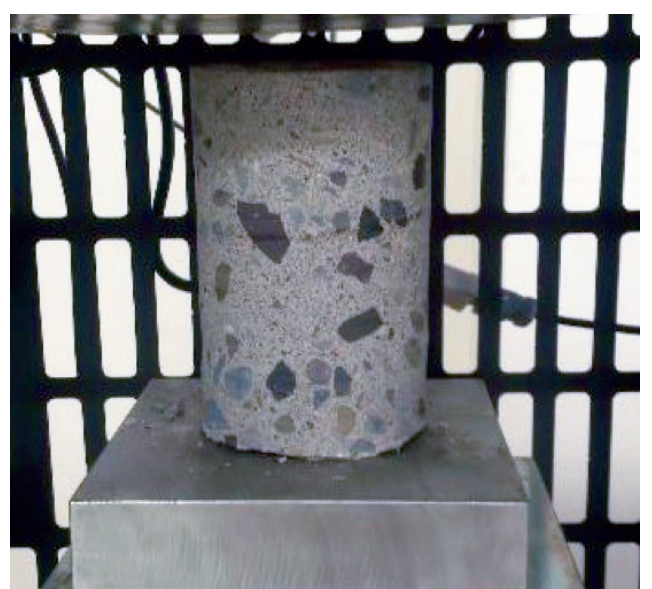

Figura 3: Ensayo de testigo de hormigón extraído del edificio en estudio

Los resultados de la Tabla 1 muestran que el módulo del edificio que presenta mayor variabilidad de la calidad del hormigón es el Módulo C, con valores de resistencia que están incluso por debajo de lo especificado en el diseño (G25), registrándose calidades tan bajas como $f^{\prime}=10 \mathrm{MPa}$ en el piso 13.

Tabla 1: Clasificación del hormigón $\left(f_{\mathrm{c}}^{\prime}\right)$ por piso según su resistencia en $\mathrm{MPa}$

\begin{tabular}{|c|c|c|c|c|}
\hline Piso & Torre A & Torre B & Torre C & Torre D \\
\hline 2 & G-25 & G-25 & G-17 & G-25 \\
\hline 3 & G-20 & G-25 & G-17 & G-20 \\
\hline 4 & G-25 & G-25 & G-25 & G-25 \\
\hline 5 & G-30 & G-25 & G-17 & G-25 \\
\hline 6 & G-25 & G-25 & G-20 & G-20 \\
\hline 7 & G-25 & G-25 & G-25 & G-25 \\
\hline 8 & G-25 & G-25 & G-20 & G-20 \\
\hline 9 & G-20 & G-10 & G-17 & G-25 \\
\hline 10 & G-25 & G-17 & G-25 & G-20 \\
\hline 11 & G-25 & G-25 & G-25 & G-25 \\
\hline 12 & G-20 & G-10 & G-20 & G-17 \\
\hline 13 & G-10 & G-20 & G-10 & G-20 \\
\hline 14 & G-17 & G-10 & G-17 & G-25 \\
\hline
\end{tabular}

\section{Modelos numéricos}

Inicialmente, el edificio fue representado mediante modelos numéricos desarrollados en el programa ETABS (2015). Los elementos vigas y columnas que en conjunto forman los marcos de hormigón, fueron modelados como elementos tipo frame, mientras que las losas y muros fueron modelados como elementos tipo shell. Se elaboró un primer conjunto de modelos numéricos considerando las propiedades mecánicas declaradas en la memoria de cálculo del proyecto. Los cuatro módulos fueron analizados independientemente, considerando la separación de $2.5 \mathrm{~cm}$ que existe entre ellos en toda su altura y desde sus fundaciones. Algunos resultados de los análisis son mostrados en la Figura 4 en la que se observa que el primer modo en los cuatro módulos corresponde al que activa el movimiento transversal del edificio. Por otra parte, las Tablas 2 a 5 muestran las frecuencias y participación modal de los 6 primeros modos de los cuatro módulos del edificio en estudio.

Sin perjuicio de lo anterior, también se elaboró un segundo modelo considerando una conexión entre los módulos, en caso de que la interfaz sísmica no haya logrado ser efectiva y exista una respuesta acoplada de todo el conjunto. El resultado de este segundo análisis se muestra en la Tabla 6. 


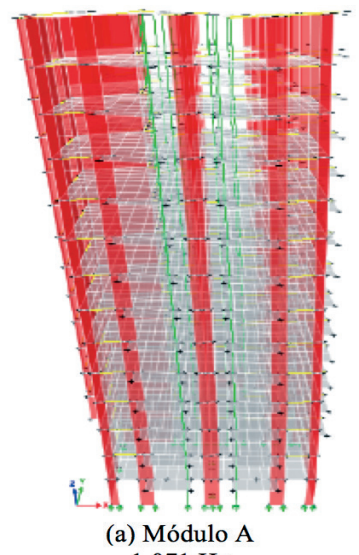

$1.071 \mathrm{~Hz}$

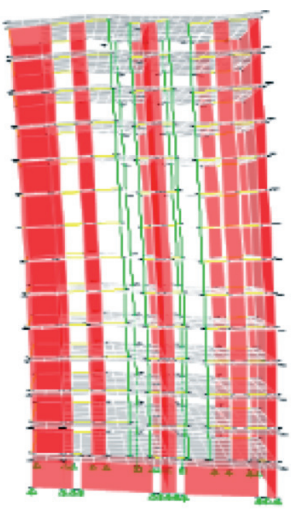

(c) Módulo C

$1.113 \mathrm{~Hz}$

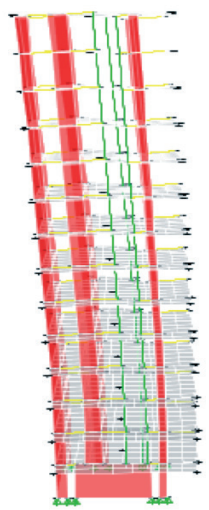

(b) Módulo B $0.873 \mathrm{~Hz}$

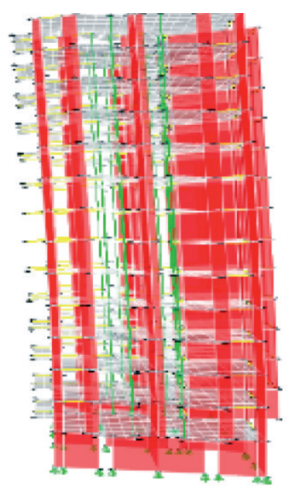

(d) Módulo D $1.125 \mathrm{~Hz}$

Figura 4: Deformada y frecuencias para el primer modo de: a) Módulo A, b) Módulo B, c) Módulo C y d) Módulo D

Tabla 2: Frecuencias y masa traslacional equivalente del Módulo A

\begin{tabular}{|c|c|c|c|}
\hline Modo & Frecuencia, $\mathrm{Hz}$ & \% modal UX & \% modal UY \\
\hline 1 & 1.072 & 68.39 & 0.00 \\
\hline 2 & 1.905 & 0.00 & 33.44 \\
\hline 3 & 2.232 & 0.00 & 35.51 \\
\hline 4 & 6.329 & 18.60 & 0.06 \\
\hline 5 & 8.000 & 0.37 & 3.41 \\
\hline 6 & 9.174 & 0.00 & 15.67 \\
\hline
\end{tabular}

Tabla 3: Frecuencias y masa traslacional equivalente del Módulo B

\begin{tabular}{|c|c|c|c|}
\hline Modo & Frecuencia, $\mathrm{Hz}$ & $\%$ modal UX & \% modal UY \\
\hline 1 & 0.873 & 62.69 & 0.13 \\
\hline 2 & 1.603 & 0.59 & 46.37 \\
\hline 3 & 2.653 & 0.33 & 18.62 \\
\hline 4 & 4.444 & 19.73 & 0.03 \\
\hline 5 & 8.475 & 0.02 & 15.03 \\
\hline 6 & 10.101 & 5.73 & 0.45 \\
\hline
\end{tabular}

Tabla 4: Frecuencias y masa traslacional equivalente del Módulo C

\begin{tabular}{|c|c|c|c|}
\hline Modo & Frecuencia, $\mathrm{Hz}$ & \% modal UX & \% modal UY \\
\hline 1 & 1.112 & 63.16 & 0.00 \\
\hline 2 & 2.169 & 0.02 & 64.73 \\
\hline 3 & 2.639 & 0.53 & 1.68 \\
\hline 4 & 5.714 & 19.77 & 0.00 \\
\hline 5 & 10.101 & 0.00 & 21.70 \\
\hline 6 & 11.494 & 0.00 & 0.02 \\
\hline
\end{tabular}

Tabla 5: Frecuencias y masa traslacional equivalente del Módulo D

\begin{tabular}{|c|c|c|c|}
\hline Modo & Frecuencia, $\mathrm{Hz}$ & $\%$ modal UX & \% modal UY \\
\hline 1 & 1.125 & 67.20 & 0.00 \\
\hline 2 & 1.949 & 0.00 & 40.37 \\
\hline 3 & 2.268 & 0.00 & 29.04 \\
\hline 4 & 6.579 & 18.55 & 0.09 \\
\hline 5 & 8.065 & 0.15 & 6.16 \\
\hline 6 & 9.346 & 0.01 & 12.94 \\
\hline
\end{tabular}

Tabla 6: Frecuencias y masa traslacional equivalente del edificio, con módulos unidos rígidamente

\begin{tabular}{|c|c|c|c|}
\hline Modo & Frecuencia, $\mathrm{Hz}$ & \% modal UX & \% modal UY \\
\hline 1 & 1.230 & 67.01 & 0.00 \\
\hline 2 & 2.108 & 0.01 & 68.13 \\
\hline 3 & 2.207 & 0.06 & 0.05 \\
\hline 4 & 6.542 & 18.69 & 0.00 \\
\hline 5 & 8.468 & 0.08 & 0.27 \\
\hline 6 & 9.123 & 0.00 & 19.68 \\
\hline
\end{tabular}

Finalmente, se elaboró un modelo numérico utilizando las propiedades del hormigón determinadas experimentalmente, observándose diferencias poco significativas en las frecuencias naturales y formas modales del sistema, tal como se evidencia en la Figura 5 y Tablas 7 a 10.

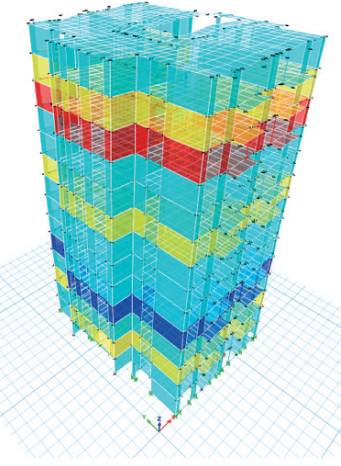

(a) Módulo A $1.065 \mathrm{~Hz}$

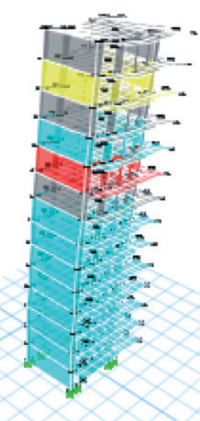

(b) Módulo B

$0.865 \mathrm{~Hz}$

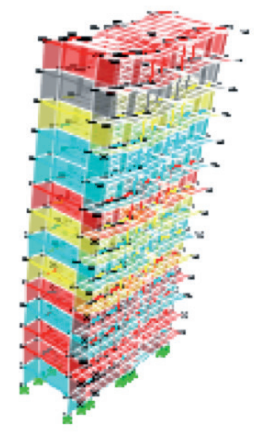

(c) Módulo C $1.031 \mathrm{~Hz}$

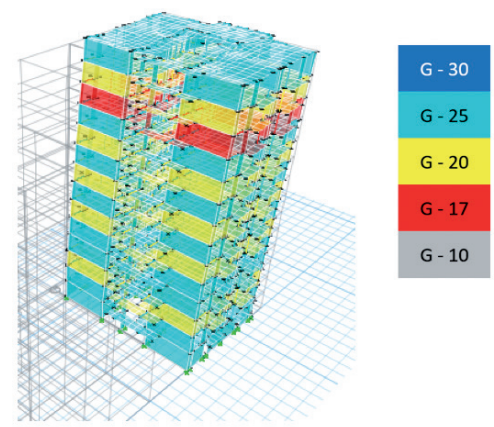

(d) Módulo D $1.109 \mathrm{~Hz}$
Figura 5: Formas y frecuencias del primer modo de vibrar del edificio considerando las propiedades experimentales del hormigón: a) Módulo A, b) Módulo B, c) Módulo C y d) Módulo D 
Tabla 9: Frecuencias y masa traslacional equivalente del Módulo $\mathrm{C}$ actualizado

\begin{tabular}{|c|c|c|c|}
\hline Modo & Frecuencia $(\mathrm{Hz})$ & \% modal UX & \% modal UY \\
\hline 1 & 1.031 & 63.29 & 0.00 \\
\hline 2 & 2.025 & 0.03 & 64.74 \\
\hline 3 & 2.459 & 0.51 & 1.55 \\
\hline 4 & 5.350 & 19.66 & 0.00 \\
\hline 5 & 9.473 & 0.00 & 21.54 \\
\hline 6 & 10.799 & 0.01 & 0.04 \\
\hline
\end{tabular}

Tabla 10: Frecuencias y masa traslacional equivalente del Módulo D actualizado

\begin{tabular}{|c|c|c|c|}
\hline Modo & Frecuencia $(\mathrm{Hz})$ & \% modal UX & \% modal UY \\
\hline 1 & 1.109 & 67.06 & 0.00 \\
\hline 2 & 1.960 & 0.00 & 38.90 \\
\hline 3 & 2.218 & 0.00 & 30.07 \\
\hline 4 & 6.422 & 18.47 & 0.09 \\
\hline 5 & 7.840 & 0.15 & 5.94 \\
\hline 6 & 9.100 & 0.01 & 13.21 \\
\hline
\end{tabular}

Estudios previos (Olivera, 2016; Cáceres, 2017; Olivera et al., 2017), lograron verificar una similitud aceptable entre la respuesta modal estimada por este modelo numérico y la respuesta real de la estructura medida mediante métodos de análisis modal operacional, especialmente en la dirección transversal sobre la cual actúan prevalentemente las cargas de viento que excitan las estructuras bajo condiciones normales de operación.

\section{Perfil bio-sísmico}

El perfil bio-sísmico es una técnica propuesta por Guendelman et al. (1997) que, mediante la evaluación de una serie de indicadores, permite detectar, en forma estimativa, posibles deficiencias en el sistema resistente de un edificio, e implementar tempranamente eventuales correcciones que mejoren su desempeño esperado, sin necesidad de elaborar modelos no-lineales de mayor complejidad y costo computacional. Para aplicar esta metodología se evalúan una serie de parámetros asociados a la rigidez, acoplamiento de modos, redundancia y ductilidad de la estructura. En este estudio estos parámetros se obtuvieron con un análisis espectral considerando un suelo Tipo C de acuerdo con lo establecido en la norma NCh433 (1996) y el DS61 (2011).

Aún cuando, los autores del método proponen la evaluación de 13 indicadores, en este estudio se consideraron solo 6, pues se estimó que ellos detectan de manera suficiente los diversos problemas de estructuración que se pesquisen en este trabajo. Los indicadores elegidos son: $i$ ) razón entre la altura total y periodo traslacional, ii) deriva de pisos en centros de gravedad, iii) desplazamiento total de techo, iv) deriva en puntos extremos, $v$ ) razón entre el periodo rotacional y periodo traslacional, vi) número de elementos sismorresistente relevantes y vii) factor de reducción espectral efectivo.

\section{Parámetro 1: Razón entre la altura total y el periodo traslacional}

Este indicador entrega información sobre la rigidez del edificio. Relaciona la altura total del edificio con el primer modo traslacional de ambas direcciones. Valores de este indicador entre $20-40 \mathrm{~m} / \mathrm{s}$ identifican a edificios flexibles, entre $40-70 \mathrm{~m} / \mathrm{s}$ los de rigidez normal y sobre 70 hasta $150 \mathrm{~m} / \mathrm{s}$ a los edificios rígidos. Los resultados de la Tabla 11 muestran que los módulos A y D tienen un indicador que clasifica el edificio como flexible en la dirección longitudinal (dir. X) y rígida en la dirección transversal (dir. $\mathrm{Y}$ ), mientras que los módulos $\mathrm{B}$ y $\mathrm{C}$ son clasificados como flexible en la dirección longitudinal (dir. X) y normales en la dirección transversal (dir. Y).

Tabla 11: Evaluación del Parámetro 1

\begin{tabular}{|c|c|c|c|c|c|}
\cline { 2 - 6 } \multicolumn{1}{c|}{} & $\begin{array}{c}\text { Periodo } \\
\mathrm{X}, \mathrm{s}\end{array}$ & $\begin{array}{c}\text { Periodo } \\
\mathrm{Y}, \mathrm{s}\end{array}$ & Altura, $\mathrm{m}$ & $\begin{array}{c}(\mathrm{H} / \mathrm{T})_{\mathrm{X}}, \\
\mathrm{m} / \mathrm{s}\end{array}$ & $\begin{array}{c}(\mathrm{H} / \mathrm{T})_{\mathrm{Y}}, \\
\mathrm{m} / \mathrm{s}\end{array}$ \\
\hline Módulo A & 0.939 & 0.453 & 34.11 & $\begin{array}{c}36.3 \\
\text { Flexible }\end{array}$ & $\begin{array}{c}75.3 \\
\text { Rígido }\end{array}$ \\
\hline Módulo B & 1.156 & 0.630 & 34.11 & $\begin{array}{c}29.5 \\
\text { Flexible }\end{array}$ & $\begin{array}{c}54.14 \\
\text { Normal }\end{array}$ \\
\hline Módulo C & 0.970 & 0.494 & 34.11 & $\begin{array}{c}35.16 \\
\text { Flexible }\end{array}$ & $\begin{array}{c}69.05 \\
\text { Normal }\end{array}$ \\
\hline $\begin{array}{c}\text { Módulo } \\
\text { D }\end{array}$ & 0.939 & 0.453 & 34.11 & $\begin{array}{c}36.3 \\
\text { Flexible }\end{array}$ & $\begin{array}{c}75.3 \\
\text { Rígido }\end{array}$ \\
\hline
\end{tabular}

\section{Parámetro 2: Deriva de entrepiso en centros de gravedad}

Este indicador mide la rigidez traslacional del edificio. La norma NCh433 (1996) limita los desplazamientos relativos entre dos pisos consecutivos (deriva de piso) medidos en el centro de gravedad de estos a un $2 \%$ de la altura de entrepiso (h). Se sugiere que este indicador no esté por debajo del $0.2 \%$ de la altura de entrepiso (Guendelman et al., 1997). Los resultados del análisis para este parámetro se muestran en la Tabla 12. Estos indican que en la dirección longitudinal del edificio (dir. X) se generan mayores desplazamientos que en la dirección transversal (dir. Y), 
lo que coincide con lo observado en el Parámetro 1. En el Módulo B y C, este indicador supera el 2\% de la altura de entrepiso prescrito como límite por la norma.

Tabla 12: Evaluación del Parámetro 2

\begin{tabular}{|c|c|c|}
\cline { 2 - 3 } \multicolumn{1}{c|}{} & Max deriva dir. X, \%o & Max deriva dir. Y, \%o \\
\hline Módulo A & 0.9 & 0.5 \\
\hline Módulo B & 2.5 & 1.5 \\
\hline Módulo C & 3.7 & 1.1 \\
\hline Módulo D & 0.9 & 0.5 \\
\hline
\end{tabular}

\section{Parámetro 3: Desplazamiento total de techo}

Este indicador, al igual que los dos anteriores, está relacionado con la rigidez traslacional del edificio. Este parámetro no está explícitamente limitado en la NCh433 (1996). Sin embargo, su valor se restringe de forma indirecta a partir de los desplazamientos de la deriva acumulada a lo largo de los pisos del edificio. Para edificios flexibles se esperan desplazamientos del nivel superior entre un 1 $2 \%$ de la altura total, mientras que, para edificios rígidos, los desplazamientos del nivel superior esperables oscilan entre un $0.2-0.5 \%$ de la altura total (Guendelman et al., 1997). La Tabla 13 muestra que el Módulo C se encuentra fuera del rango "normal", mientras que los demás módulos se encuentran dentro del rango llamado "normal".

Tabla 13: Evaluación del parámetro 3

\begin{tabular}{|c|c|c|}
\cline { 2 - 3 } \multicolumn{1}{c|}{} & $\begin{array}{c}\text { Max desplazamiento } \\
\text { total dir. X, \% }\end{array}$ & $\begin{array}{c}\text { Max desplazamiento } \\
\text { total dir. Y, \%o }\end{array}$ \\
\hline Módulo A & 0.7 & 0.4 \\
\hline Módulo B & 1.8 & 1.1 \\
\hline Módulo C & 2.7 & 0.8 \\
\hline Módulo D & 0.7 & 0.4 \\
\hline
\end{tabular}

\section{Parámetro 4: Deriva de entrepiso en puntos extremos}

Este parámetro que estima el grado de rigidez rotacional del edificio está explícitamente definido en la NCh433 (1996), donde se indica que los desplazamientos adicionales en cualquier punto de la planta, generados por efecto de traslación y rotación de los diafragmas de piso, no deben superar en más de un 1\%o el desplazamiento de entrepiso medido en los centros de masa de ese nivel. La Tabla 14 muestra los resultados para cada uno de los módulos. En todos los casos se observa que este parámetro no supera el $1 \%$, por lo que se podría esperar que el comportamiento torsional fuese poco significativo.
Tabla 14. Evaluación del parámetro 4

\begin{tabular}{|c|c|c|}
\cline { 2 - 3 } \multicolumn{1}{c|}{} & $\begin{array}{c}\text { Deriva en puntos } \\
\text { extremos dir. X, \%o }\end{array}$ & $\begin{array}{c}\text { Deriva en puntos } \\
\text { extremos dir. Y, \%o }\end{array}$ \\
\hline Módulo A & 0.06 & 0.22 \\
\hline Módulo B & 0.39 & 0.65 \\
\hline Módulo C & 0.30 & 0.28 \\
\hline Módulo D & 0.06 & 0.22 \\
\hline
\end{tabular}

\section{Parámetro 5: Razón entre el periodo rotacional y el periodo traslacional}

Este indicador estima el grado de acoplamiento entre modos de vibrar traslacionales y rotacionales. En edificios adecuadamente estructurados se espera que la razón entre ambos periodos se aleje en al menos un $20 \%$ de la unidad. Los resultados mostrados en la Tabla 15 muestran que existe acoplamiento entre la torsión y la traslación en la dirección transversal (Dir. Y) para los Módulos A y D.

Tabla 15: Evaluación del parámetro 5

\begin{tabular}{|c|c|c|c|c|c|}
\cline { 2 - 6 } \multicolumn{1}{c|}{} & $\begin{array}{c}\text { Periodo } \\
\text { traslacional } \\
\text { X Tx, s }\end{array}$ & $\begin{array}{c}\text { Periodo } \\
\text { traslacional } \\
\text { Y, Ty, } \mathrm{s}\end{array}$ & $\begin{array}{c}\text { Periodo } \\
\text { rotacional } \\
\mathrm{Tr}, \mathrm{s}\end{array}$ & $\mathrm{Tr} / \mathrm{Tx}$ & $\mathrm{Tr} / \mathrm{Ty}$ \\
\hline Módulo A & 0.939 & 0.453 & 0.453 & 0.48 & 1.00 \\
\hline Módulo B & 1.156 & 0.63 & 0.381 & 0.33 & 0.60 \\
\hline Módulo C & 0.97 & 0.494 & 0.407 & 0.42 & 0.82 \\
\hline Módulo D & 0.939 & 0.453 & 0.453 & 0.48 & 1.00 \\
\hline
\end{tabular}

\section{Parámetro 6: Números de elementos sismorresistentes relevantes}

Este indicador estima la capacidad que posee la estructura para traspasar los esfuerzos generados por un sismo a sus elementos resistentes en cada dirección. Se calcula sumando el número de elementos verticales, responsables de resistir acciones sísmicas en el nivel basal, tales como muros y columnas, en una dirección determinada. Se recomienda que el número de ejes resistentes sea siempre mayor a tres, para asegurar la redundancia estructural (Guendelman et al., 1997). De forma similar se puede calcular la densidad de muros en cada dirección como la razón entre la sumatoria del área transversal de muros en una dirección determinada respecto al área total de la planta. Las Figuras 6 a 9 muestran las plantas típicas de cada uno de los módulos, donde se pueden identificar el número de elementos resistentes y calcular la densidad de muros en cada dirección. Los resultados de este cálculo se presentan en la Tabla 16. 
Tabla 16: Evaluación del parámetro 6

\begin{tabular}{|c|c|c|c|c|c|c|c|}
\hline & $\begin{array}{c}\text { Superficie total } \\
\text { de la planta, } \mathrm{m}^{2}\end{array}$ & $\begin{array}{c}\text { Cantidad de } \\
\text { elementos en } \\
\text { dir X }\end{array}$ & $\begin{array}{c}\text { Cantidad de } \\
\text { elementos en } \\
\text { dir Y }\end{array}$ & $\begin{array}{c}\text { Superficie de } \\
\text { muros en } \\
\text { dir. X, } \mathrm{m}^{2}\end{array}$ & $\begin{array}{c}\text { Superficie de } \\
\text { muros en } \\
\text { dir. Y, } \mathrm{m}^{2}\end{array}$ & $\begin{array}{c}\text { Densidad de } \\
\text { muros en dir. X }\end{array}$ & $\begin{array}{c}\text { Densidad de } \\
\text { muros en dir. Y }\end{array}$ \\
\hline Módulo A & 294 & 4 & 3 & 9.92 & 6.09 & 0.034 & 0.021 \\
Módulo B & 78 & 2 & 2 & 1.71 & 1.94 & 0.022 \\
Módulo C & 125 & 2 & 3 & 4.11 & 2.90 & 0.033 \\
Módulo D & 294 & 4 & 3 & 9.92 & 6.09 & 0.034 \\
\hline
\end{tabular}

a)

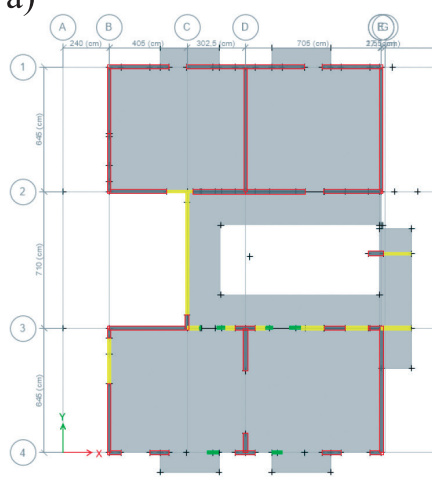

b)

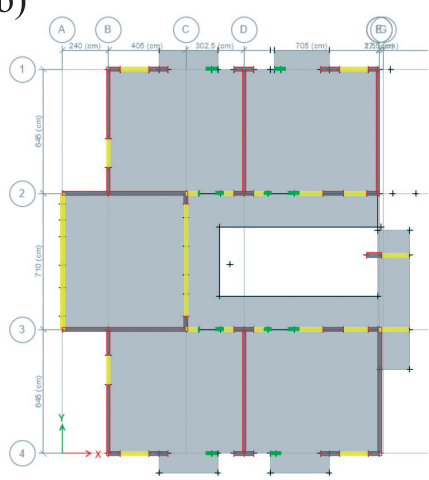

Figura 6: Plantas típicas. Módulo A, a) primer y b) segundo piso

a)
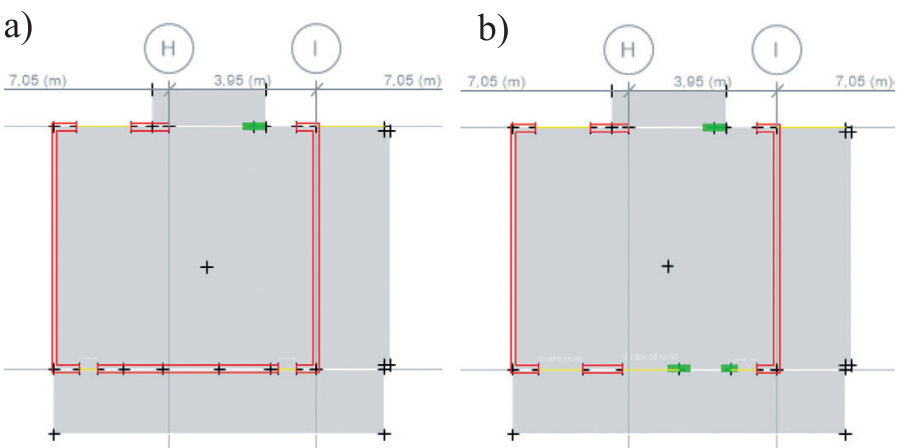

Figura 7: Plantas típicas. Módulo B, a) primer y b) segundo piso

a)

b)

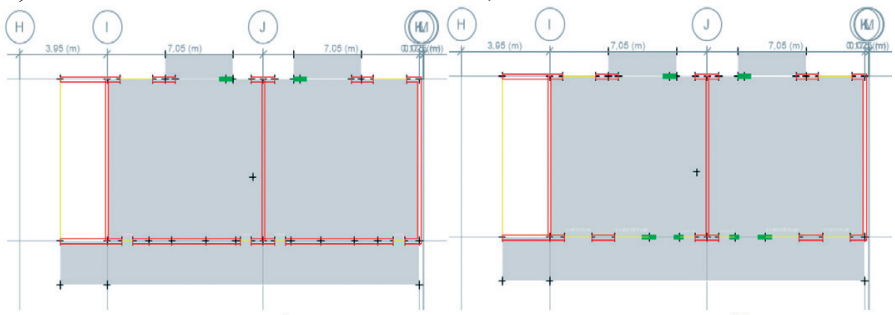

Figura 8: Plantas típicas. Módulo C, a) primer y b) segundo piso

\section{Parámetro 7: Factor de reducción espectral efectivo, $\mathrm{R}^{* *}$}

Este indicador permite estimar el grado de ductilidad que requiere el edificio. Un $\mathrm{R}^{* *}$ muy pequeño, indica que a)

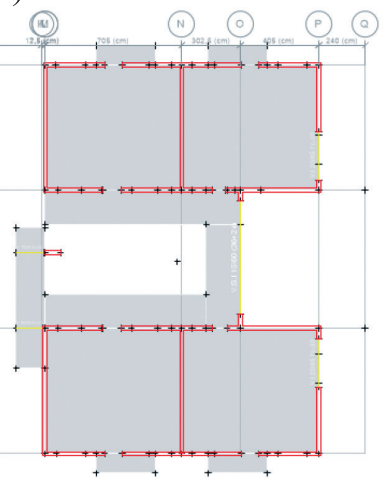

b)

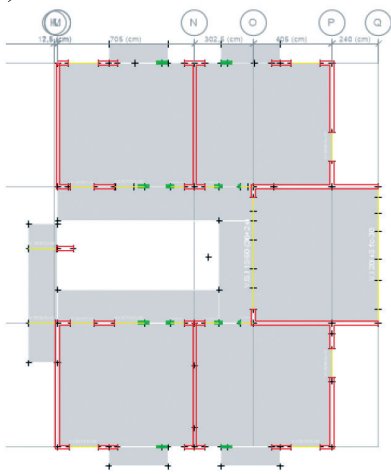

Figura 9: Plantas típicas. Módulo D, a) primer y b) segundo piso

en su diseño fue aplicado un espectro inelástico con una reducción mínima. En tanto, un $\mathrm{R}^{* *}$ elevado, indicaría que el diseño se realizó con un espectro inelástico muy reducido. Para valores de $\mathrm{R}^{* *}$ comprendidos entre tres y siete se recomienda que se complemente el análisis normativo con procedimientos no lineales aproximados tipo push-over y que, para valores superiores a siete, sea imprescindible utilizar técnicas más refinadas (Guendelman et al., 1997). Este indicador está dado por la ecuación (1). Los resultados del análisis para este parámetro se muestran en la Tabla 17. Estos indican que en cada uno de los módulos este parámetro se encuentra entre tres y siete, en ambas direcciones.

$$
R^{* *}=\frac{R^{*}}{1.4 f_{\min } f_{\max }}
$$

en donde $\mathrm{R}^{*}$ es el factor de reducción de la aceleración espectral, $f_{\min }$ es el factor de amplificación por corte mínimo y es $f_{\max }$ el factor de reducción por corte máximo.

Finalmente, en la Tabla 18 se presenta un resumen de los resultados de los análisis realizados mediante el método del perfil bio-sísmico. De este análisis se pueden esperar para el Módulo A clasificaciones divergentes 
entre la dirección longitudinal y transversal (flexible y rígido, respectivamente) y un posible acoplamiento entre la traslación y la rotación. En el caso del Módulo $\mathrm{B}$ encontramos clasificaciones dentro del rango normal a excepción del parámetro 6 , ya que este módulo posee dos ejes resistentes en la dirección longitudinal y transversal. Para el Módulo C la clasificación es muy similar al Módulo $\mathrm{B}$, encontramos clasificaciones dentro del rango normal y al evaluar el parámetro 6 encontramos que en la dirección longitudinal posee dos ejes resistentes. En el caso del Módulo D este presenta la misma clasificación que el Módulo A. Al evaluar el parámetro 7 este indicaría que el análisis normativo no es suficiente para el diseño de esta estructura y que se debiese complementar con procedimientos no lineales aproximados tipo push-over.

Tabla 17: Evaluación del parámetro 7

\begin{tabular}{|l|c|c|c|c|c|c|}
\cline { 2 - 7 } \multicolumn{1}{c|}{} & $\mathrm{T}^{*},{ }_{\mathrm{x}}, \mathrm{s}$ & $\mathrm{T}_{\mathrm{y}}^{*} \mathrm{~s}$ & $\mathrm{R}^{*}{ }_{\mathrm{x}}$ & $\mathrm{R}^{*} \mathrm{y}$ & $\mathrm{R}^{* *}{ }_{\mathrm{x}}$ & $\mathrm{R}^{* *}{ }_{\mathrm{y}}$ \\
\hline Módulo A & 0.939 & 0.453 & 8.49 & 4.35 & 6.06 & 3.10 \\
\hline Módulo B & 1.156 & 0.630 & 4.82 & 6.68 & 3.44 & 4.77 \\
\hline Módulo C & 0.970 & 0.494 & 5.42 & 6.82 & 3.87 & 4.87 \\
\hline Módulo D & 0.939 & 0.453 & 8.49 & 4.35 & 6.06 & 3.10 \\
\hline
\end{tabular}

\section{Análisis dinámico incremental}

Para completar este estudio se realizó un análisis dinámico incremental (Vamvatsikos y Cornell, 2002). En este tipo de estudios se desarrollan sucesivos análisis no lineales tiempo historia utilizando registros sísmicos escalados a distintos niveles de intensidad de movimiento sísmico. En este caso, se utilizó el registro sísmico del terremoto de Maule $2010 \operatorname{con} M_{\mathrm{w}}=8.8$ (Figura 10) medido en el centro de la ciudad de Concepción en una estación con suelo denso $\left(V_{\mathrm{s} 30} \geq 350 \mathrm{~m} / \mathrm{s}\right)$, cuya duración significativa fue de $85 \mathrm{~s}$ y la máxima aceleración de suelo alcanzó los 0.42 g a los $20.4 \mathrm{~s}$. Se realizaron 11 análisis con este registro, incrementando su intensidad progresivamente un $10 \%$ cada vez hasta duplicarla.

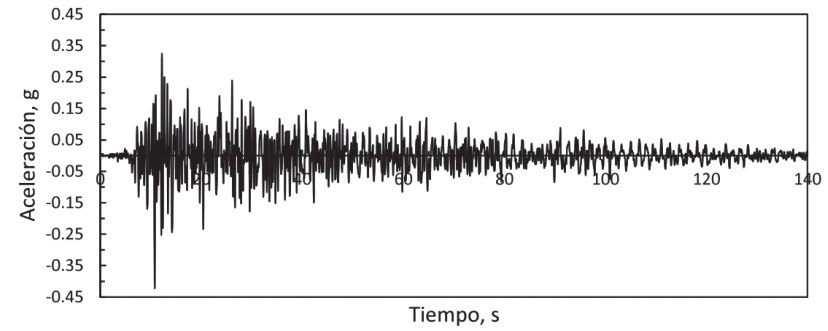

Figura 10: Registro de aceleraciones de Concepción, terremoto del Maule $2010\left(M_{\mathrm{w}}=8.8\right)$

Tabla 18: Resumen perfil bio-sísmico

\begin{tabular}{|c|c|c|c|c|c|c|c|c|}
\hline & \multicolumn{2}{|c|}{ Módulo A } & \multicolumn{2}{|c|}{ Módulo B } & \multicolumn{2}{|c|}{ Módulo C } & \multicolumn{2}{|c|}{ Módulo D } \\
\hline Parámetros & Dir X & Dir Y & Dir X & Dir Y & $\operatorname{Dir} X$ & Dir Y & Dir X & Dir Y \\
\hline $\begin{array}{l}\text { Parámetro 1: } \\
\text { Razón altura vs periodo } \\
\text { traslacional }\end{array}$ & Flexible & Rígido & Flexible & Normal & Flexible & Normal & Flexible & Rígido \\
\hline $\begin{array}{l}\text { Parámetro 2: } \\
\text { Deriva centros de } \\
\text { gravedad }\end{array}$ & Normal & Normal & $\begin{array}{c}\text { Fuera de } \\
\text { rango }\end{array}$ & Normal & $\begin{array}{l}\text { Fuera de } \\
\text { rango }\end{array}$ & Normal & Normal & Normal \\
\hline $\begin{array}{l}\text { Parámetro 3: } \\
\text { Desplazamiento total } \\
\text { nivel superior }\end{array}$ & Normal & Normal & Normal & Normal & $\begin{array}{l}\text { Fuera de } \\
\text { rango }\end{array}$ & Normal & Normal & Normal \\
\hline $\begin{array}{l}\text { Parámetro 4: } \\
\text { Deriva puntos extremos }\end{array}$ & Normal & Normal & Normal & Normal & Normal & Normal & Normal & Normal \\
\hline $\begin{array}{l}\text { Parámetro 5: } \\
\text { Razón periodo } \\
\text { rotacional vs Periodo } \\
\text { traslacional }\end{array}$ & Normal & $\begin{array}{c}\text { Fuera de } \\
\text { rango }\end{array}$ & Normal & Normal & Normal & Normal & Normal & $\begin{array}{c}\text { Fuera de } \\
\text { rango }\end{array}$ \\
\hline $\begin{array}{l}\text { Parámetro 6: } \\
\text { Número de elementos } \\
\text { relevantes en la } \\
\text { resistencia sísmica }\end{array}$ & Normal & Normal & $\begin{array}{c}\text { Fuera de } \\
\text { rango }\end{array}$ & $\begin{array}{c}\text { Fuera de } \\
\text { rango }\end{array}$ & $\begin{array}{l}\text { Fuera de } \\
\text { rango }\end{array}$ & Normal & Normal & Normal \\
\hline $\begin{array}{l}\text { Parámetro 7: } \\
\text { Factor de reducción } \\
\text { espectral efectivo, } \mathrm{R}^{* *}\end{array}$ & $\begin{array}{c}\text { Fuera de } \\
\text { rango }\end{array}$ & $\begin{array}{c}\text { Fuera de } \\
\text { rango }\end{array}$ & $\begin{array}{c}\text { Fuera de } \\
\text { rango }\end{array}$ & $\begin{array}{c}\text { Fuera de } \\
\text { rango }\end{array}$ & $\begin{array}{l}\text { Fuera de } \\
\text { rango }\end{array}$ & $\begin{array}{c}\text { Fuera de } \\
\text { rango }\end{array}$ & $\begin{array}{c}\text { Fuera de } \\
\text { rango }\end{array}$ & $\begin{array}{c}\text { Fuera de } \\
\text { rango }\end{array}$ \\
\hline
\end{tabular}


Se usó elementos de plasticidad concentrada para modelar los elementos de muros y marcos (puntos de color blanco en Figura 11). Se consideraron largos de rótulas plásticas de un $50 \%$ de la altura para muros y en un $10 \%$ y $90 \%$ del largo para los elementos de marcos. La degradación de resistencia y rigidez de estos elementos siguió una ley histerética del tipo propuesta por Takeda et al. (1970), cuya envolvente quedó determinada por los parámetros establecidos en ASCE/SEI 41-13 (2013) que define los puntos $\mathrm{A}, \mathrm{B}$ y $\mathrm{C}$ de la curva de respuesta momento-giro de mostrados en la Figura 12. Dado que no se contaba con información respecto a la distribución de armadura de cada elemento estructural, se optó por considerar cuantías mínimas uniformemente distribuidas en cada uno de los elementos. Para realizar el diagnóstico de la estructura se hizo un análisis de la deformación angular entre los segmentos a ambos lados de las rótulas plásticas de los elementos sismos resistentes del edificio y de los desplazamientos relativos de los centros de gravedad entre pisos consecutivos. Esto es, variable de la respuesta a nivel local y global, respectivamente. El análisis antes descrito se aplicó únicamente al Módulo A, que presenta una mayor variabilidad de la calidad del hormigón y que presentó parámetros del perfil bio-sísmico fuera de los rangos propuestos por Guendelman et al. (1997).

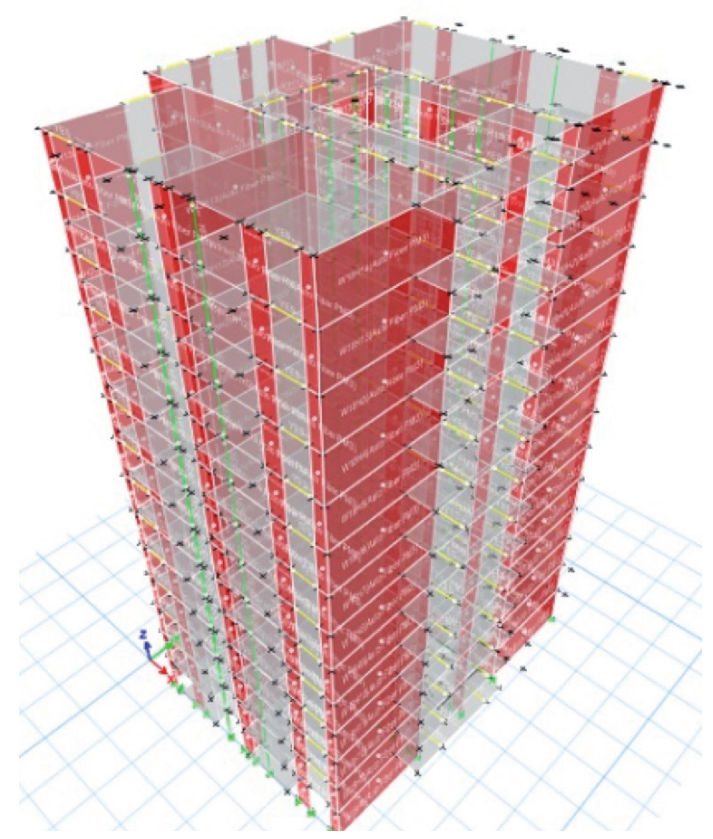

Figura 11: Asignación de rótulas plásticas, Módulo A

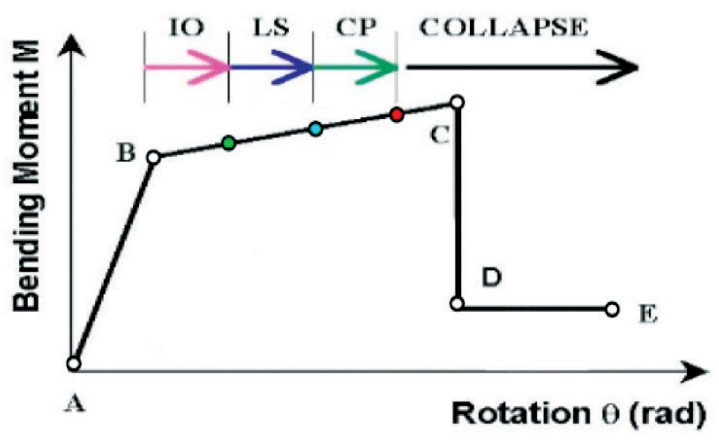

Figura 12: Curva de respuesta momento-giro de rótulas plásticas, elementos de hormigón armado (Brzev y Sherstobitoff, 2004)

\section{Análisis local de la estructura}

La Figura 12 muestra la curva de respuesta momento-giro que describe el comportamiento de las rótulas plásticas en elementos de hormigón armado. Los puntos que se observan en dicha figura representan distintas condiciones de deformación tras aplicación de carga, donde A corresponde a la condición sin carga, el punto B representa el inicio de la fluencia, el punto $\mathrm{C}$ corresponde a la máxima capacidad de carga y $\mathrm{D}$ es el esfuerzo residual que representa la disminución de resistencia que puede estar asociada a la fractura del refuerzo longitudinal, mientras que $\mathrm{E}$ es el punto de máxima deformación.

Por otro lado, los tramos designados como IO, LS y CP corresponden a los diferentes niveles de desempeño de los elementos en función de la degradación de rigidez y/o resistencia que se espera observar en los edificios. Estos niveles de desempeño se definen de la siguiente forma:

- Ocupación inmediata (IO): Posterior a la ocurrencia de un evento sísmico, la estructura se mantiene en operación. Los elementos estructurales mantendrán su funcionamiento en el rango elástico. Los elementos no estructurales y el equipamiento no sufrirán daños. El umbral superior de rotación de las rótulas para este nivel de desempeño es $0.002 \mathrm{rad}$.

- Seguridad a la vida (LS): Los elementos estructurales sufren daños localizados debido al evento sísmico, que pueden ser reparados. Este es el nivel de rendimiento esperado en edificios diseñados según códigos modernos ante sismos severos. El umbral superior de rotación de las rótulas para este nivel de desempeño es $0.003 \mathrm{rad}$. 
- Prevención al colapso (CP): Los daños que se observan en la estructura afectan elementos resistente importantes y no son reparables. Sin embargo, la edificación no llega a colapsar. El umbral superior de rotación de las rótulas para este nivel de desempeño es $0.004 \mathrm{rad}$.

El análisis desarrollado indica que las rotaciones en los elementos tipo viga no superarían el límite LS (0.003 rad) en ningún caso y, además, las rotaciones en las columnas y los muros no superarían el límite IO (0.002 rad) en los niveles de trancamiento de la estructura. Por lo tanto, el Módulo A presentaría un comportamiento sísmico deseable bajo las condiciones de los análisis y frente al registro analizado.

\section{Análisis global de la estructura}

La norma chilena NCh 433 (1996) establece que el desplazamiento relativo máximo entre dos pisos consecutivos, medidos en el centro de masas en cada dirección de análisis, no debe ser mayor que la altura entrepiso multiplicada por 0.002 . Este límite puede ser considerado como un umbral de desempeño operacional de ocupación inmediata IO, pues anticipa poco daño de los elementos no estructurales. En las Figuras 13 y 14, se muestran los desplazamientos de entrepiso para cada nivel y dirección de análisis. En dichas tablas se observa que en todos los casos el desplazamiento relativo entre pisos supera el 2\%, lo que significa que el edificio incursiona en el nivel de desempeño de seguridad de vida.

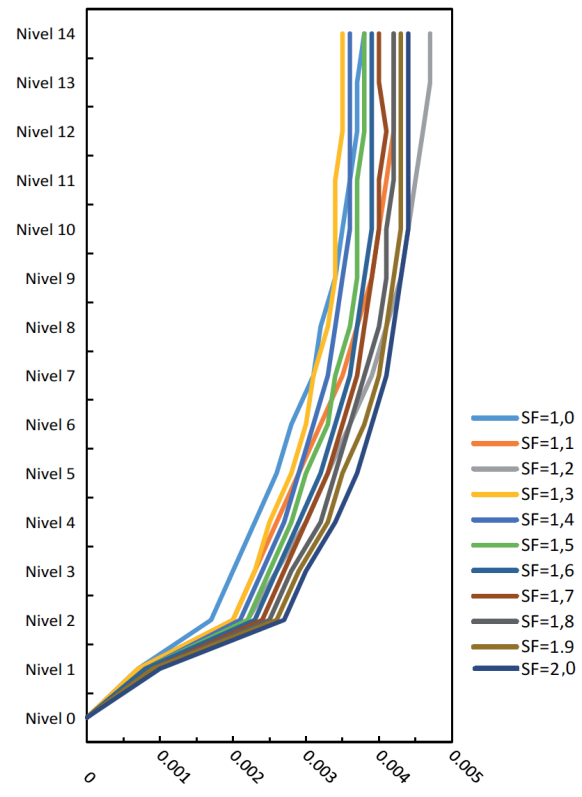

Figura13: Desplazamientos de entrepiso dir. $\mathrm{X}$
No obstante, lo anterior, es importante hacer notar que al evaluar los desplazamientos laterales en dirección longitudinal (dir. X), incluso en el caso del registro sísmico sin amplificar $(\mathrm{PGA}=0.42 \mathrm{~g}$ ), se registran desplazamientos que superan el permitido por la junta de expansión sísmica $(2.5 \mathrm{~cm})$, con un desplazamiento máximo de $9.15 \mathrm{~cm}$, tal como se muestra en la Tabla 19, por lo que se deberían esperar problemas de colisión entre los módulos.

Tabla 19: Máximo desplazamiento dir. X utilizando registro sísmico sin incremento

\begin{tabular}{|c|c|c|}
\hline Nivel & $(+)$ UX, cm & $(-)$ UX, cm \\
\hline 14 & 8.15 & -9.15 \\
\hline 13 & 7.46 & -8.38 \\
\hline 12 & 6.77 & -7.59 \\
\hline 11 & 6.07 & -6.81 \\
\hline 10 & 5.37 & -6.03 \\
\hline 9 & 4.68 & -5.25 \\
\hline 8 & 4.00 & -4.49 \\
\hline 7 & 3.33 & -3.74 \\
\hline 6 & 2.68 & -3.01 \\
\hline 5 & 2.06 & -2.32 \\
\hline 4 & 1.48 & -1.66 \\
\hline 3 & 0.95 & -1.06 \\
\hline 2 & 0.47 & -0.53 \\
\hline 1 & 0.12 & -0.12 \\
\hline
\end{tabular}

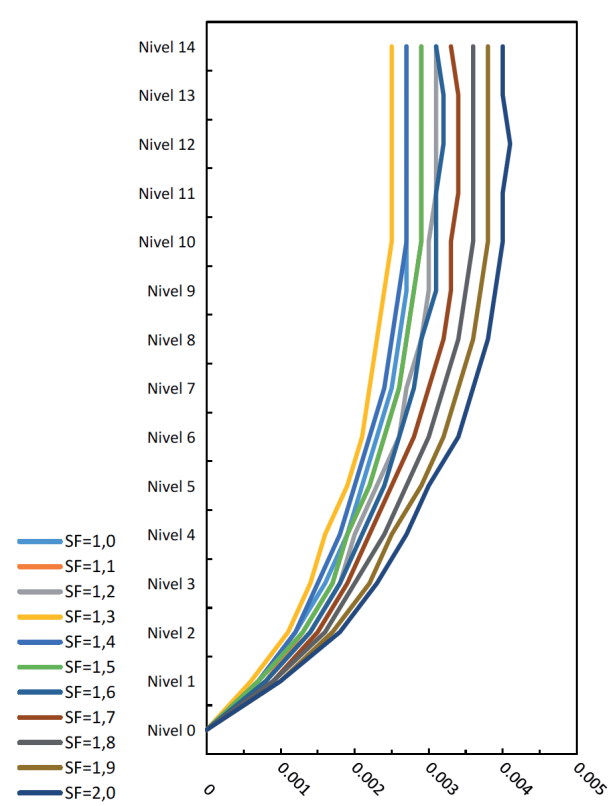

Figura14: Desplazamientos de entrepiso dir. Y 


\section{Conclusiones}

En este estudio se presentó la evaluación global de la "salud estructural" de un edificio habitacional de 14 pisos, potencialmente vulnerable a daños debido a terremotos. La evaluación estructural se realizó combinando ensayos destructivos y no-destructivos. Utilizando la información sobre la calidad del hormigón obtenida experimentalmente se generó un modelo numérico lineal del edificio, el cual fue utilizado para evaluar la salud global del edificio mediante una metodología llamada perfil bio-sísmico y un análisis dinámico incremental.

La evaluación experimental del hormigón identificó cinco calidades diferentes. El hormigón de calidad más baja corresponde a un G-10 $\left(f_{\mathrm{c}}^{\prime}=10 \mathrm{MPa}\right)$, muy por debajo de la calidad especificada para este proyecto $\left(\mathrm{G}-25, f_{\mathrm{c}}{ }_{\mathrm{c}}=\right.$ $25 \mathrm{MPa}$ ). Si bien, este ensayo se realizó únicamente sobre losas y no se desarrolló en muros, los resultados levantan una señal de alerta respecto a la calidad de los materiales utilizados y el control de calidad durante la construcción de la obra. Estos resultados deficientes no pueden atribuirse a problemas localizados, pues los resultados se repitieron en diferentes ubicaciones de la losa.

En lo que respecta al perfil bio-sísmico, se espera que el edificio en general cumpla con las disposiciones normativas chilenas, a excepción de los Módulos B y C en la dirección longitudinal que superan el límite de desplazamiento entrepiso impuesto por la norma sísmica chilena y que no condicionan la respuesta global del Módulo B y C. Sin embargo, el Módulo A y D presentan indicadores de rigidez distintos entre la dirección longitudinal y transversal (flexible y rígido, respectivamente) además de un acoplamiento entre la traslación y la rotación. Por lo tanto, estos módulos presentan parámetros que indican que la estructuración fue deficitaria, todo lo cual pudo haberse corregido con una detección oportuna en la etapa de diseño. En este sentido, el perfil bio-sísmico es un buen método que permite alertar el correcto o mal comportamiento global que pudiesen tener las estructuras sin necesidad de implementar modelos de mayor complejidad analítica. Sin embargo, este método no es capaz de anticipar fallas locales en los elementos resistentes de la estructura, tal como lo hace, por ejemplo, el análisis incremental dinámico utilizado en este estudio.
Al evaluar el desempeño estructural del Módulo A mediante el análisis dinámico incremental con un registro del evento sísmico de $M_{\mathrm{w}}=8.8$ de Maule 2010, se encontró que a pesar de que los desplazamientos entrepiso indican que se supera el límite impuesto por la norma sísmica chilena, este no es un indicador de daño del edificio, ya que estos umbrales de desplazamiento están asociados a las condiciones operacionales de desempeño. Sin embargo, los desplazamientos máximos para el registro sísmico sin incrementar en la dirección longitudinal, superan el desplazamiento permitido por la junta de expansión sísmica de $2.5 \mathrm{~cm}$. Por lo tanto, frente a un evento sísmico severo se esperarían posibles colisiones entre los módulos del edificio. A nivel local los elementos estructurales presentarían un buen desempeño, pues las rótulas plásticas de los elementos estructurales alcanzaron rotaciones que no exceden el umbral superior del rango de Seguridad de Vida (0.003 rad).

\section{Agradecimientos}

Se agradece la colaboración de los Ingenieros Civiles Leonel Vergara, Cristóbal Salamanca, Miguel Vega y Patricio Cáceres, que a través de sus proyectos de titulación contribuyeron en el desarrollo de este estudio. Se agradece también el importante aporte del Dr. Patricio Quintana Gallo quien con su experiencia y punto de vista ayudó a mejorar la calidad de este artículo.

\section{Referencias}

ASCE/SEI 41-13 (2013). Seismic evaluation and retrofit of existing buildings. ASCE Standard. American Society of Civil Engineers, Reston, Virginia, USA

ASTMC805 (2018). Standard test method for rebound number of hardened concrete. ASTM International, West Conshohocken, PA, USA

Brzev, S. and Sherstobitoff, J. (2004). Performance-based seismic evaluation of concrete reservoir structures. $13^{\text {th }}$ World Conference on Earthquake Engineering, Vancouver, Canada, Paper No. 1823

Cáceres, P. (2017). Caracterización dinámica de un edificio residencial de hormigón armado. Proyecto de título de Ingeniero Civil. Universidad Católica de la Santísima Concepción, Concepción, Chile 
DS61 (2011). Decreto supremo que modifica el diseño sísmico de edificios. Ministerio de la Vivienda y Urbanismo MINVU. Diario Oficial de la República de Chile

ETABS (2015). Integrated Building Design Software ETABS Nonlinear version 15. Reference Manual. Computer and Structures, Inc. CSI, Walnut Creek, California, USA

Guendelman, T., Guendelman, M. y Lindenberg, J. (1997). Perfil bio-sísmico de edificios. VII Jornadas Chilenas de Sismología e Ingeniería Antisísmica ACHISINA y I Congreso Iberoamericano de Ingeniería Sísmica, La Serena, Chile

NCh170 (2016). Hormigón - Requisitos generales, referidas a dosis mínima de cemento. Instituto Nacional de Normalización INN, Santiago, Chile

NCh1565 (2009). Hormigón - Determinación de índice esclerométrico. Instituto Nacional de Normalización INN, Santiago, Chile

NCh1171 (2012). Hormigón - Testigos de hormigón endurecido, extracción y ensayos. Instituto Nacional de Normalización INN, Santiago, Chile

NCh1037 (2009). Hormigón - Ensayo de compresión de probetas cúbicas y cilíndricas. Instituto Nacional de Normalización INN, Santiago, Chile

NCh433 (1996). Diseño sísmico de edificios. Modificada el 2009. Instituto Nacional de Normalización INN, Santiago, Chile INN
Olivera, J.J. (2016). Caracterización dinámica de un edificio de hormigón armado basado en un análisis modal operacional. Proyecto de título de Ingeniero Civil. Universidad Católica de la Santísima Concepción, Concepción, Chile

Olivera, J.J., Vergara, L. and Oyarzo, C. (2017). Structural health assessment of a $\mathrm{R} / \mathrm{C}$ building in the coastal area of Concepción, Chile. Procedia Engineering 199, 2214-2219

Peeters, B. and Ventura, C.E. (2003). Comparative study of modal analysis techniques for bridge characteristics. Mechanical Systems and Signal Processing 17(5), 965-988

Vamvatsikos, D. and Cornell, C.A. (2002). Incremental dynamic analysis. Earthquake Engineering and Structural Dynamics 31(3), 491-514

Ventura, C.E. and Horyna, T. (1997). Structural assessment by modal analysis in Western Canada. 15 th International Modal Analysis Conference, Society for Experimental Mechanics, Orlando, Florida, USA, 234-239

Wilson, A.W., Abdul Karim, A.R., Oyarzo-Vera, C., Omenzetter, P. and Ingham, J.M. (2008). Dynamic testing of a timber floor diaphragm in an unreinforced masonry building. $14^{\text {th }}$ World Conference on Earthquake Engineering. Beijing, China, 1-8 